\title{
A case study of mutual current effect on circulating current protection system
}

\author{
Kevin Gausultan Hadith Mangunkusumo ${ }^{1, *}$, Aristo Adi Kusuma ${ }^{1, *}$, and Buyung Sofiarto Munir ${ }^{1}$
}

${ }^{1}$ PLN Research Institute, Transmission and Distribution Department, Jakarta

\begin{abstract}
Mutual impedance generated by the double circuit lines become such a challenge to be solved. Mutual induction arises when transmission line is a parallel line on a common right of way. In this study, a system model to observed mutual current effect on circulating current protection (CCP) system are explained. The system model is validated using actual recorded fault current waveform. In one and half breaker substation, the mutual current in maintenance line may cause CCP to pick up.
\end{abstract}

\section{Introduction}

The complexity of power system development, boost the use of double circuit transmission line. Parallel transmission line is utilized to enhance the reliability and security of energy transfer [1]. On the other hand mutual impedance generated by the double circuit lines become such a challenge to be solved.

Mutual induction arises when transmission line is a parallel line on a common right of way [2]. The problem of mutual impedance happens when unbalanced faults occur on one line causing induced current and voltage in the double circuit transmission line [3]. The problem becomes more complicated when the lines are operated at different voltages such as in one of the line is under maintenance condition [4]. Even if the parallel lines were fully transposed, it can only minimize the positive and negative sequence mutual impedance but the zero sequence mutual impedance is still present [5]. The mutual current generated by mutual impedance may cause distance relay under or overreach [6]. The influence of the zero sequence mutual impedance is about $70 \%$ of the zero sequence self-impedance when the parallel lines are mounted on the same tower [7]. The influence of the mutual current on circulating current protection system based on real case condition is explained in this study.

The rest of the paper is organized as follows. Section II explains the circulating current protection (CCP) working principle in general. Section III, the system fault based on actual fault condition is described. Section IV, the system model is conducted and some assumptions is detailed. Section V are simulation and discussion. Finally, a conclusion is given in the last Section.

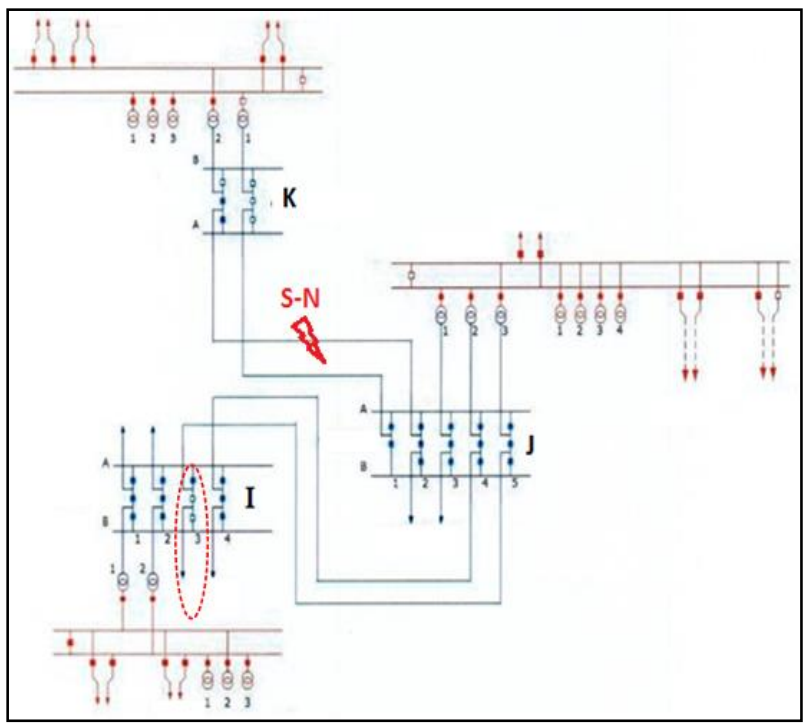

Fig. 1. Fault condition on double circuit transmission lines.

\section{System fault condition}

Fig. 1 shows the fault condition on the existing system single line diagram. The $500 \mathrm{kV}$ substations system are represented by H-I-J-K substation. The substations system are using one and half breaker configuration. One phase to neutral fault happened on one of the line between substation $\mathrm{K}$ and substation $\mathrm{J}$. The fault was recorded by distance relay in substation J. Based on the fault locator, the fault was about $4 \mathrm{~km}$ from substation $\mathrm{J}$. The distance relay tripped the circuit breaker A2 and $\mathrm{AB} 2$ at substation $\mathrm{J}$. The relay indicator showed zone 1 $\mathrm{S}-\mathrm{N}$ fault condition. As the main protection system, the distance relay sent the DTT signal to the substation K to instantaneous tripping the circuit breaker A2 and AB2 (substation K). In the existing data, the fault has done isolated by the main protection coordination. However, the Circulating Current Protection (CCP) in substation I

\footnotetext{
* Corresponding author: kevin.mangunkusumo@pln.co.id
} 
has also been triggered by the fault. As shown in fig 2, line I-H2 (substation I bay3) was off the grid before the fault happened. The line $\mathrm{I}-\mathrm{H} 2$ was in maintenance condition thus the disconnector on both substation I and substation $\mathrm{H}$ were opened and grounded on both side. Even though line I-H2 was off the grid, the fault was assumed initiating CCP at Substation I to picked up.

\section{Circulating current protection}

Circulating Current Protection (CCP) is used as the diameter protection in one and half breaker Substation. $\mathrm{CCP}$ is used to protecting area between Current Transformer (CT) at bay line and CT at circuit breaker in between diameter (AB: $\mathrm{B}$ or $\mathrm{AB}$ : $\mathrm{A}$ as shown in Fig. 1). When the fault happen inside the CCP working area, the relay will be triggered and tripping 2 circuit breaker (AB: $\mathrm{B}$ or $\mathrm{AB}: \mathrm{A}$ ) then sending direct transfer trip (DTT) signal to the opposite substation [8]. Therefore, if the CT bay line and CT diameter read different value and the value is more than the setting then the CCP will pick up.

CCP is also known as Merz Price Connection, which using Differential Relay working principle [9]. Fig. 2 shows the single line diagram of the one and half breaker substation configuration with CCP wiring diagram as diameter protection. Each diameter is protected by 2 CCP (main CCP and secondary CCP as a backup).

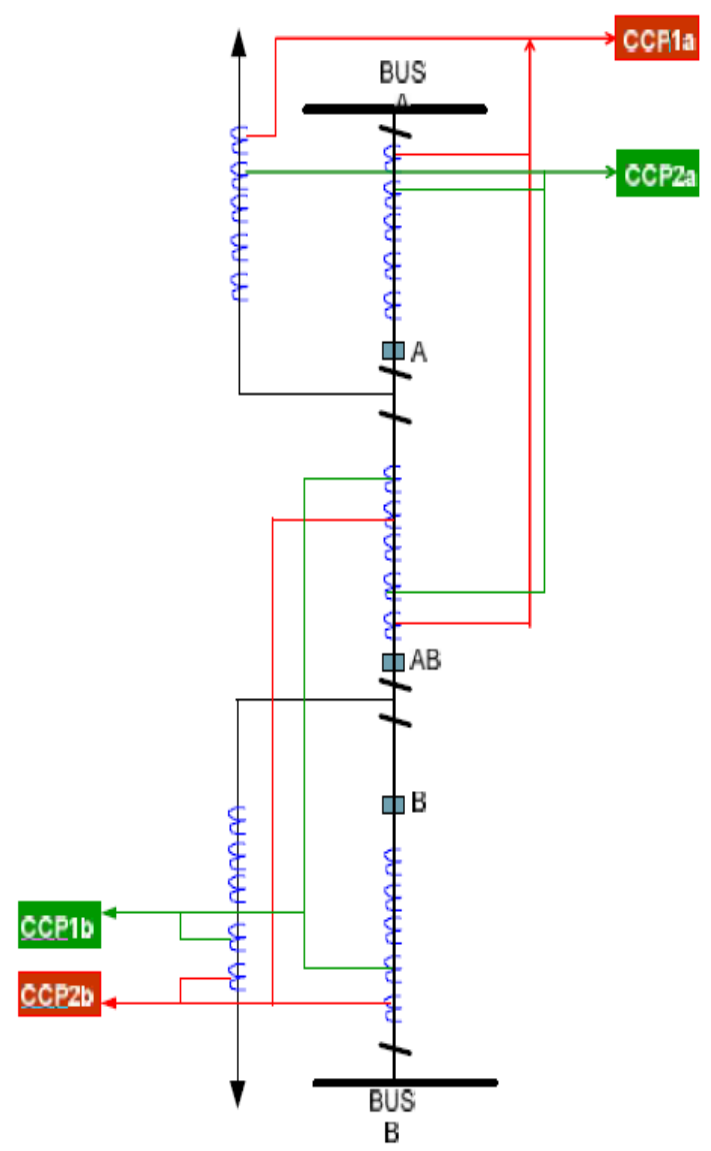

Fig. 2. Fault condition on double circuit transmission lines.

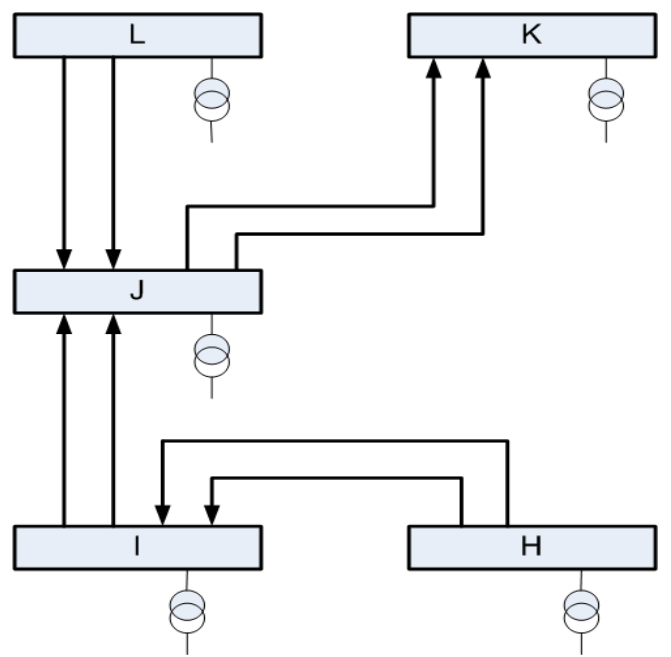

Fig. 3. The system power flow diagram.

\section{Discussion}

Simulation Model is based on power flow diagram shows in the Fig. 3 the power flow diagram is the real existing condition before the fault occur. As shown in Fig. 3 Substation $\mathrm{K}$ is supplied from substation J, however substation $\mathrm{J}$ is supplied from substation $\mathrm{H}$ and L. Thus, there are 2 sources, substation $\mathrm{H}$ and substation L. Below the substation $\mathrm{H}$ and $\mathrm{L}$ is simplified as the power source. The length of the line is using the real existing data and the geometry of the $500 \mathrm{kV}$ tower is typical. The fault is occur in the line J-K2 at $4 \mathrm{~km}$ from substation J. The system model is done using Transient Analysis software. Some assumtions are used to simulate the overall system model:

- The model is only observed $500 \mathrm{kV}$ High Voltage Transmission Line $\mathrm{H}-\mathrm{I}-\mathrm{J}-\mathrm{K}-\mathrm{L}$

- Transmission line is assumed as straight flat track with typical $500 \mathrm{kV}$ tower

- The transmission model is using LCC Bergeron type

- The conductor is using Aluminium Conductor Steel Reinforce (ACSR) DOVE.

Fig. 4 shows the overall system model in this study. Line $\mathrm{I}-\mathrm{H} 2$ is under maintenance condition, thus the disconnectors are open and grounded on both side on line I-H2 while on the line I-H1 was flowing short circuit current because of phase to ground fault on line J-K2.

\section{Simulation result and discussion}

Fig. 5 shows the fault current recorded by Digital Fault Recorder (DFR) on line I-H2. The R, S and T current signal shows almost the same phase angle and phase $\mathrm{S}$ have the highest magnitude. The recorded fault current will be used to validate the system model. Fig. 6 shows the mutual current signal generated by the simulation model. The signal is taken on line I-H2. The red circle 


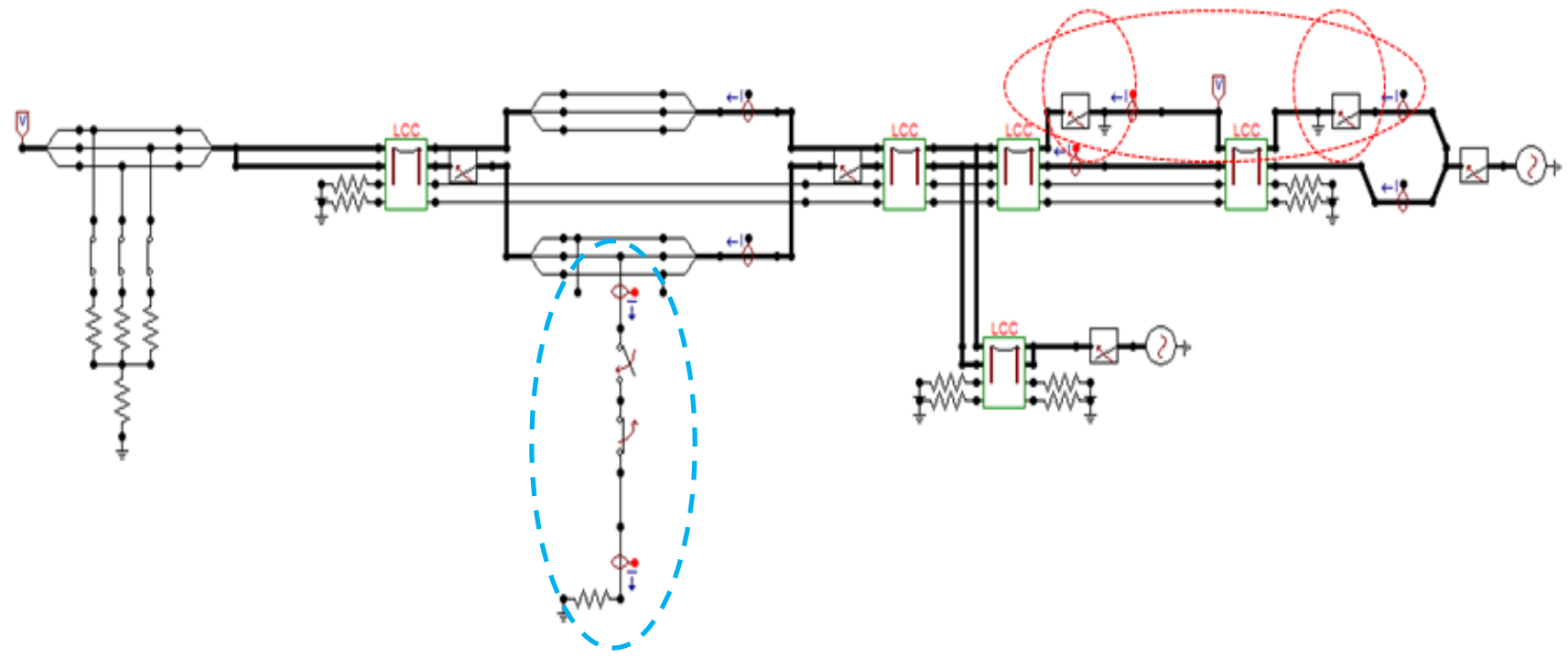

Fig. 4. The overall system model.

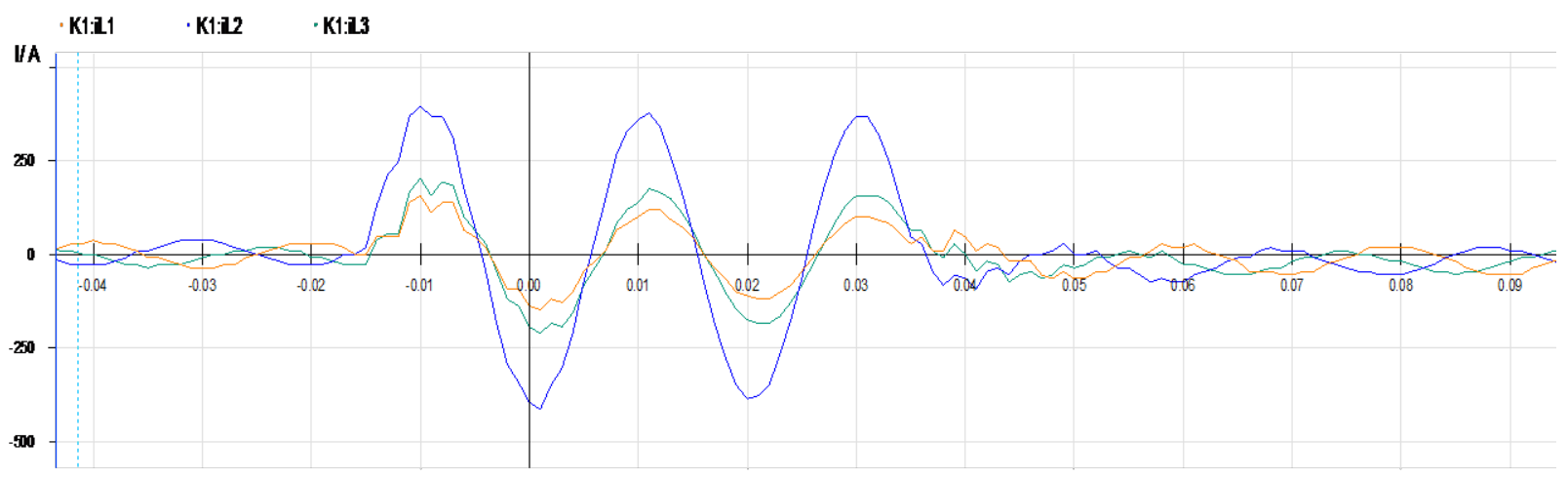

Fig. 5. Actual recorded DFR signal.

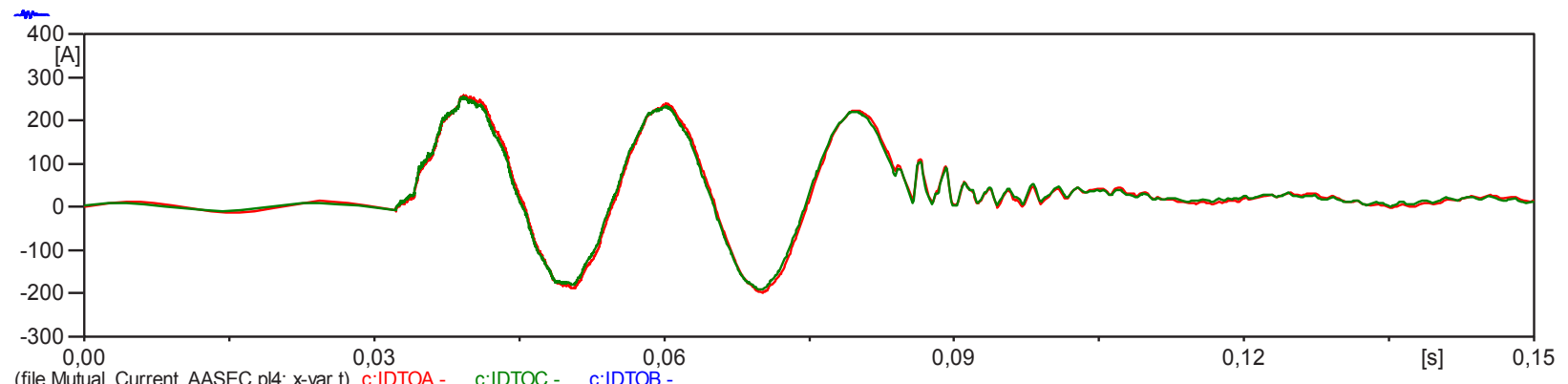

Fig. 6. Mutual current simulation result.

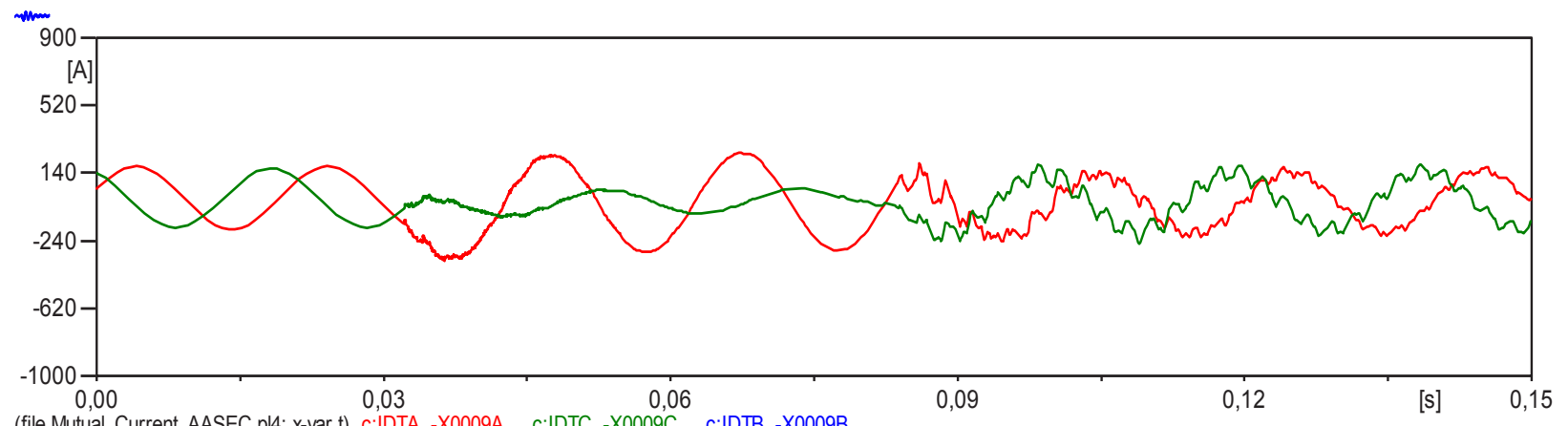

(file Mutual_Current_AASEC.p14; $x$-var t) c:IDTA -X0009A C:IDTC -X0009C c:IDTB -X0009B

Fig. 7. Short circuit current on line J-K2. 
Shown in Fig. 4 shows that the disconnector open and grounded on both side, thus the line I-H2 is off the grid. The simulation result shows the same trend with the recorded fault current. As shown in Fig. 5 and Fig. 6, the current signal R, S, T both have almost the same phase angle and phase $\mathrm{S}$ both have the highest magnitude. Therefore, the simulation model has been done validated by comparing the recorded signal and the simulation result.

Fig. 7 shows the short circuit current phase $S$ to ground simulation result. The short circuit current is taken on line I-H1. The fault simulation is done by short circuit phase $\mathrm{S}$ to ground on line $\mathrm{J}-\mathrm{K} 2$ as shown by blue circle in Fig. 4. Therefore on the double circuit transmission line, when short circuit happen on one of the line, mutual current will be generated on the opposite line even if the line is off the grid or open and grounded on both side (usually in maintenance condition).

Fig. 8 shows the substation I wiring diagram at bay 3-line I-H2. From the figure, the detail position of the $\mathrm{CT}$ are founded. The CT position especially on line I-H2 is located after the disconnector B3-3 as shown by red circle. Thus, the position of the $\mathrm{CT}$ is allowing the $\mathrm{CT}$ to recording the fault current on line $\mathrm{I}-\mathrm{H} 2$ even if the disconnector is open. In this study case, the line is off the grid which means the disconnector of the line are open and grounded on both side but the CT keep recording the

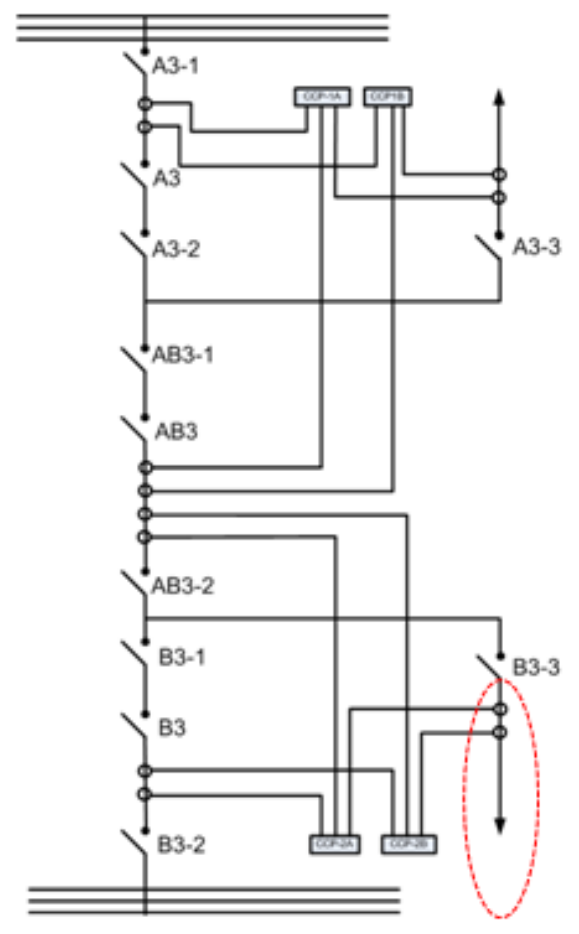

Fig. 8. The system power flow diagram.

Fault current. The CCP as the diameter protection is using differential relay principle. CCP will compare the recorded current on the line $\mathrm{I}-\mathrm{H} 2$ which recorded by $\mathrm{CT}$ placed after the B3-3 with $\mathrm{CT}$ between $\mathrm{AB} 3-2 \mathrm{AB} 3$ and $\mathrm{CT}$ between B3:B3-2. Because of the fault, current is only generated on the line I-H2 and doesn't flows through the diameter, the difference recorded current value will triggered the CCP to pick up.

\section{Conclusion}

In this paper, mutual current effect on CCP system has been explained. The system model is based on real system and validated using actual recorded fault current.On the parallel line transmission system, the mutual current can be generated on the line under maintenance condition/off the grid (one line of two parallel lines which disconnector open and grounded on both sides) when phase to ground fault happen on the opposite line. In one and half breaker configuration, the mutual current in maintenance line may cause CCP to pick up.

\section{References}

1. Z. Hu, X. Zhongzhu, J. Fan and M. Chen, "Novel Method of Live Line Measuring the Zero Sequence Parameters of Transmission Lines with Mutual Inductance," presented at IEEE Power \& Energy Society General Meeting, (2009).

2. J. Holbeck I, L. J and Marting, "The Effects of Mutual Induction Between Parallel Transmission Lines on Current Flow to Ground Faults," AIEE Trans., vol. 62, no. 12, p. 11, (1943).

3. C. Li, Z. Hu, M. Jiang and Z. Zhang, "New Method of Measuring the Zero Sequence Parameters of Double-Circuit EHV/UHV Transmission Lines," presented at Power and Energy Engineering Conference (APPEEC), Shanghai, (2012).

4. K. R. Pillay and B. S. Rigby, "Studying the impact of mutual coupling on distance," presented at The Falls Resort and Conference Centre, Livingstone, Zambia, (2011).

5. F. Calero, "Mutual Impedance in Parallel Lines Protective Relaying Problems and Principles," presented at Annual Western Protective Relay Conference, Washington, (1991).

6. I. C. Borascu dan S. S. Iliescu, "Influence of the Zero Sequence Mutual Impedance to the Distance Protection," UPB Scientific Bulletin, vol. 77, no. 4, p. Series C, (2015).

7. G. E. Alexander and J. G. Andrichak, Ground Distance Relaying Problems and Principles, Washington, (1991).

8. J. L. Blackburn, Symmetrical Components for Power Systems Engineering, (CRC Press, Ohio, 1989).

9. L. C. W. Frerk, Power System Protection 3: Application, chap 13, (1995). 